\title{
Effective gas-phase diffusion coefficient in soils
}

\author{
C.-S. Fen \\ Department of Environmental Engineering and Science, \\ Feng Chia University, Taichung, Taiwan, Republic of China
}

\begin{abstract}
Determination of the effective gas-phase diffusion coefficient is important to quantify gas diffusion for a number of environmental pollution problems involving migration and release of hazardous gases or organic vapors in soils. Conventionally, the only considered transport mechanism for this coefficient is molecular diffusion. This study additionally considers the effects of Knudsen diffusion and nonequimolar flux on this coefficient and proposes a relationship of this coefficient with both molecular and Knudsen diffusion coefficients, ratio of molar masses and composition of component gases for gas diffusion in soils. Through investigating calculation results of this relationship for hypothetical diffusion systems having different soil permeabilities and diffusing gases at a range of water saturation levels, it is found that the Knudsen and nonequimolar effects can only be substantial for systems with dry soil permeabilities less than $10^{-13} \mathrm{~m}^{2}$ at any saturation. The Knudsen effect on this relationship is further demonstrated through comparing results obtained from this relationship to the measured effective diffusion coefficients for 72 soil cores, which all have high silt and clay contents, given in the literature.

Keywords: gas diffusion in soil, molecular diffusion, Knudsen diffusion, diffusibility.
\end{abstract}

\section{Introduction}

Volatile organic compounds (VOCs) are common groundwater contaminants threatening our environment. Volatile contaminants can quickly spread in the subsurface by vaporization at dissolved or free phase and by gas phase transport through soil pores. Gas diffusion is an important transport mechanism controlling migration and distribution of volatile contaminants. Other environmental pollution problems such as emissions of methane, $\mathrm{CO}_{2}$ or organic 
vapors from landfills [20], emissions of fumigant and pesticide [14] and migration of naturally generated radon through soils and foundation materials [16] can also be dominated by gas diffusion. Besides, mass of volatile chemicals can naturally be reduced through gas diffusion in contaminated soils. Therefore, an accurate quantification of gas diffusion becomes important for assessment of natural attenuation, remediation and monitoring network design and risk assessment of contaminated sites.

Fick's first law of diffusion is conventionally applied to compute diffusive flux of a gas component in soils. In the application, the air-filled gas-phase diffusion coefficient is modified by a diffusibility factor, leading to the so-called effective diffusion coefficient, to account for the impedance to diffusion caused by the tortuous nature of pores in soils. Determination of the diffusibility factor is important in predicting the magnitude of gas diffusion, and thereby delineating other transport and fate processes in soils.

In the past, numerous experimental studies proposed various relationships of the diffusibility factor with soil porosity, air-filled porosity and some empirical constants, e.g., [5, 8, 22, 26]. Millington and Quirk [18], Ball [4], Friejer [10] and Schaefer et al. [24] treated the porous structure of soil as joint tubes and developed theoretical models for this factor. Van Duin [27] and Lahvis et al. [17], however, used experimental and/or field data to determine the diffusibility factor as functions of air-filled porosity and several fitting constants. Moldrup et al. [19] and Weerts et al. [30] assessed the dependence of this factor on soil water characteristic parameters of disturbed and undisturbed soils.

A rigorously constitutive relationship of component gas diffusive flux in soils is described by the Dusty Gas Model (DGM) equations [7]. The component diffusive flux for multicomponent gas diffusion in soils can be a Fickian expression only under specific conditions. For example, Jaynes and Rogowski [12], Amali et al. [2] and Webb and Pruess [29] mentioned a Fickian expression is valid only for a binary gas diffusion system with a trace gas and for a ternary gas diffusion system with a stagnant gas. Fen and Abriola [9] used the DGM equations to obtain the component diffusive flux in a Fickian form for a two-component gas transport system with gas components of equal molecular masses under isothermal and isobaric conditions. A correction factor, called the obstruction factor, is defined in the DGM, to account for the obstruction of soil particles to molecular diffusion. Brugeman [6], Neale and Nader [21] and AbuEl-Sha'r and Abriola [1] applied the DGM theory and conducted equimolar countercurrent diffusion experiments in porous systems to determine this factor as functions of air-filled porosity. Abu-El-Sha'r and Abriola [1] also determined the Knudsen diffusion coefficient for single gas flow systems with the same soil samples as the ones used for determining the obstruction factor in twocomponent gas diffusion experiments. However, the Knudsen effect is not pronounced in their diffusion experiments, as a result that the diffusibility factor is equivalent to the obstruction factor.

It is shown that Fick's law is generally applied to gas diffusion in soils. A lot of experiments have been done to find the relationships among the effective diffusion coefficient, air-filled molecular diffusion coefficient, total and air-filled 
porosities of soil. Seldom has considered the coupling effect of molecular and Knudsen diffusions in low permeability soil systems and the nonequimolar effect due to unequal molecular masses of the diffusing and ambient gases on gas diffusion. For the application of Fick's law to the interpretation of laboratory data for gas diffusion in soil columns, two questions need to be clarified: (1) what is the specific soil condition under which Knudsen diffusion is substantial and should be considered in the effective diffusion coefficient? (2) How significant is the nonequimolar effect on gas diffusion in soil systems?

This study intends to resolve these questions by conducting a series of laboratory experiments and assessing the dependence of the diffusibility factor on the Knudsen diffusion coefficient, gas component and composition. These experiments are being undertaken and aim at independently determining the obstruction factor for two-component gas diffusion and the permeability and Knudsen diffusion coefficients for single gas flow in low permeability soil systems with the same soil samples. A literature survey of existing correlations of the effective diffusion coefficient was also carried out and the corresponding experimental data was used to analysis the relative importance of molecular and Knudsen diffusions and the nonequimolar effect. This paper presents this study result.

\section{Materials and methods}

Without considering surface, temperature diffusions and the gravitational effect, the DGM equations are expressed as

$$
\sum_{j=1, j \neq i}^{n} \frac{X_{i} N_{j}^{D}-X_{j} N_{i}^{D}}{Q_{m} D_{i j}^{o}}-\frac{N_{i}^{D}}{D_{i}^{K}}=\frac{1}{R T} \nabla p_{i} \quad i=1,2, \ldots ., n
$$

in which $n$ is the total number of gas components in the gas mixture; $X_{i}$ is the mole fraction of component $i ; \hat{N}_{i}^{D}$ is the total diffusive flux of component $i$ [mol $\left.\mathrm{m}^{-2} \mathrm{~s}^{-1}\right] ; T$ is the absolute temperature $\left[{ }^{\circ} \mathrm{K}\right] ; R$ is the universal gas constant $[\mathrm{kg}$ $\left.\mathrm{m}^{2} \mathrm{sec}^{-2} \mathrm{~K}^{-1} \mathrm{~mol}^{-1}\right] ; p_{i}$ is the partial pressure of component $i\left[\mathrm{~kg} \mathrm{~m}^{-3} \mathrm{~s}^{-2}\right] ; D_{i j}^{o}$ is the air-filled binary molecular diffusion coefficient of gas pair $i$ and $j\left[\mathrm{~m}^{2} \mathrm{sec}^{-1}\right] ; Q_{m}$ is the obstruction factor; and $D_{i}^{K}$ is the Knudsen diffusion coefficient of component $i\left[\mathrm{~m}^{2} \mathrm{sec}^{-1}\right]$. Thorstenson and Pollock [25] provided the following relationship for $D_{i}^{K}$,

$$
D_{i}^{K}=\frac{k_{e} b_{i}}{\mu_{i}}
$$

where $k_{e}$ is the gas effective permeability $\left[\mathrm{m}^{2}\right]$ and $k_{e}=k k_{r g}$ in which $k$ is the intrinsic permeability $\left[\mathrm{m}^{2}\right]$ and $k_{r g}$ is the gas relative permeability; $\mu_{i}$ is the 
dynamic viscosity of component $i\left[\mathrm{~kg} \mathrm{~m}^{-1} \mathrm{~s}^{-1}\right] ; b_{i}$ is the Klinkenberg parameter [Pa]. According to [23], $b_{i}$ can be determined by the following relationship

$$
b_{i}=5.57 k_{e}^{-0.24} \frac{\mu_{i}}{\mu_{\text {air }}} \sqrt{\frac{M_{\text {air }}}{M_{i}}}
$$

where $M_{\text {air }}$ and $M_{i}$ are molar masses of air and component $i\left[\mathrm{~kg} \mathrm{~mol}^{-1}\right]$.

According to [9], for a binary system of components $A$ and $B$ under isobaric condition, the total diffusive flux for each component can be written explicitly by manipulation of eqn (1):

$$
N_{A}^{D}=-\frac{Q_{m} D_{A B}^{o} D_{A}^{K} C}{Q_{m} D_{A B}^{o}+D_{A}^{K}\left(1-a_{A} X_{A}\right)} \nabla X_{A}
$$

where $C=\sum C_{i}$ is the molar concentration of the gas mixture $\left(\mathrm{mol} \mathrm{m}^{-3}\right)$; $a_{A}=1-\left(M_{A} / M_{B}\right)^{1 / 2}$.

When both components have equal molecular masses in the system, the flux is expressed as

$$
N_{A}^{D}=-\left(\frac{1}{D_{A}^{K}}+\frac{1}{Q_{m} D_{A B}^{o}}\right)^{-1} C \nabla X_{A}
$$

Fick's law of diffusion applied to gas diffusion in soil systems is expressed as

$$
N_{i}^{D}=-Q D_{i j}^{o} \nabla C_{i}
$$

where $Q$ is the diffusibility factor and is generally used to account for the impedance of soil particle to gas diffusion. A comparison of eqns (4) and (6) reveals that the diffusibility factor defined in Fick's law of diffusion can be composition-dependent and is related to the obstruction factor as follows:

$$
Q=\frac{Q_{m} D_{A}^{K}}{Q_{m} D_{A B}^{0}+D_{A}^{K}\left(1-a_{A} X_{A}\right)}
$$

Eqn (7) can be further simplified to become: (1) $Q=\frac{Q_{m} D_{A}^{K}}{Q_{m} D_{A B}^{0}+D_{A}^{K}}$, for the system with equal molecular mass components $\left(\boldsymbol{a}_{\boldsymbol{A}}=0\right)$ or involving diffusion of a dilute gas in any gas mixture ( $\boldsymbol{X}_{A} \approx 0$ ); (2) $Q=\frac{D_{A}^{K}}{D_{A B}^{0}}$, for gas transport dominated by Knudsen diffusion $\left(\boldsymbol{D}_{\boldsymbol{A}}^{\boldsymbol{K}}<<\boldsymbol{D}_{A B}^{\boldsymbol{o}}\right.$ ); (3) $\boldsymbol{Q}=\boldsymbol{Q}_{\boldsymbol{m}}$, for gas transport dominated by molecular diffusion $\left(\boldsymbol{D}_{\boldsymbol{A}}^{K} \gg>\boldsymbol{D}_{A B}^{o}\right)$. 
The relationship shown in eqn (7) reveals that independent experiments for assessing both the obstruction factor and the Knudsen diffusion coefficient for the same soil sample are required to verify the dependence of the diffusibility factor on these two parameters. Obviously, these experiments need to be conducted in low permeability soil columns and different diffusing gases should be considered to investigate the nonequimolar effect.

To identify the relative importance of molecular and Knudsen diffusions under a variety of soil conditions, the diffusibility is assessed for a series of hypothetical gas diffusion systems. The diffusibility factor is calculated according to eqn (7) with neglecting the $X_{A}$ term (i.e. assuming diffusing gas is dilute in these hypothetical systems) and compared to the obstruction factor, which is calculated based on Millington and Quirk relationship. Soil systems with different permeabilities and diffusing gases at different levels of water saturation were considered in the calculation. Note that the Knudsen diffusion coefficient is related to gas permeability, which depends on soil type and water saturation, i.e., eqns (2) and (3).

In this study, the measured results of the diffusibility factor for different gas diffusion experiments, specifically, in low permeability soils, were collected from literature, which include those presented in $[3,4,10,11,13,15,28,31]$, for totally 72 soil cores, which all have sand content less than $15 \%$. Different gas components were used as a diffusing gas in these experiments. Air is used as an ambient gas. For most of these diffusion experiments, soil or gas permeability was not measured. For assessing the Knudsen effect on gas diffusion in these soil systems, an empirical relationship of soil permeability with soil composition is used in this study

$$
k=\frac{1}{m}\left[\frac{\left(1-\theta_{T}\right)^{2}}{\theta_{T}{ }^{3}}\left(\frac{\alpha}{100} \sum \frac{f_{i}}{d_{m}}\right)^{2}\right]^{-1}
$$

where $\theta_{T}$ is the total porosity of the soil sample; $m$ is a packing factor determined experimentally to be about $5 ; \alpha$ is a sand-shape factor varying from 6 for spherical grains to 7.7 for angular grains; $f_{i}$ is the fraction of sand held between adjacent sieves; and $d_{m}$ is the geometric mean of the rated size of adjacent sieves. Note that eqn (8) does not work well for the porous medium composed of finegrained material.

\section{Results and discussion}

The diffusibility factor was calculated based on eqns (2), (3) and (7) for several hypothetical diffusion systems, in which $\mathrm{CO}, \mathrm{TCE}$ or $\mathrm{CH}_{4}$ is considered as a diffusing gas and $\mathrm{N}_{2}$ is an ambient gas. This calculation also considers a range of soil permeabilities from $10^{-10}$ to $10^{-16} \mathrm{~m}^{2}$ and water saturation levels between 0.4 and 0.8 . The obstruction factor was calculated based on [18]. A reduction of gas permeability due to partial saturation of soil is evaluated according to Brook and Corey's relationship. Figure 2 presents the percentage difference between 
the calculated values of the diffusibility and the obstruction factors related to water saturation of soil and the corresponding soil effective permeability. The difference is minimal for the diffusion systems with dry soil permeabilities greater than $10^{-13} \mathrm{~m}^{2}$. This implies the Knudsen effect can be neglected for gas diffusion in soil systems within this dry soil permeability and the tested saturation ranges. For soil systems with dry soil permeabilities less than $10^{-13} \mathrm{~m}^{2}$, the difference starts to be significant, which is $6 \%$ at least and $75 \%$ at most for these studied cases. Soils in this permeability range have high silt and clay contents. Besides, the diffusibility values are less than those of the obstruction factor when considering the Knudsen effect in the diffusibility. Different diffusing gas also makes the difference changed even though the gas is dilute in the system. For all of the studied cases, the difference for the $\mathrm{CO} / \mathrm{N}_{2}$ system is the greatest and for the $\mathrm{CH}_{4} / \mathrm{N}_{2}$ system is the least. As dry soil permeability decreases, discrepancies of the difference between different diffusing gas systems also increase. Note that the difference does decrease as the soil water saturation increases.
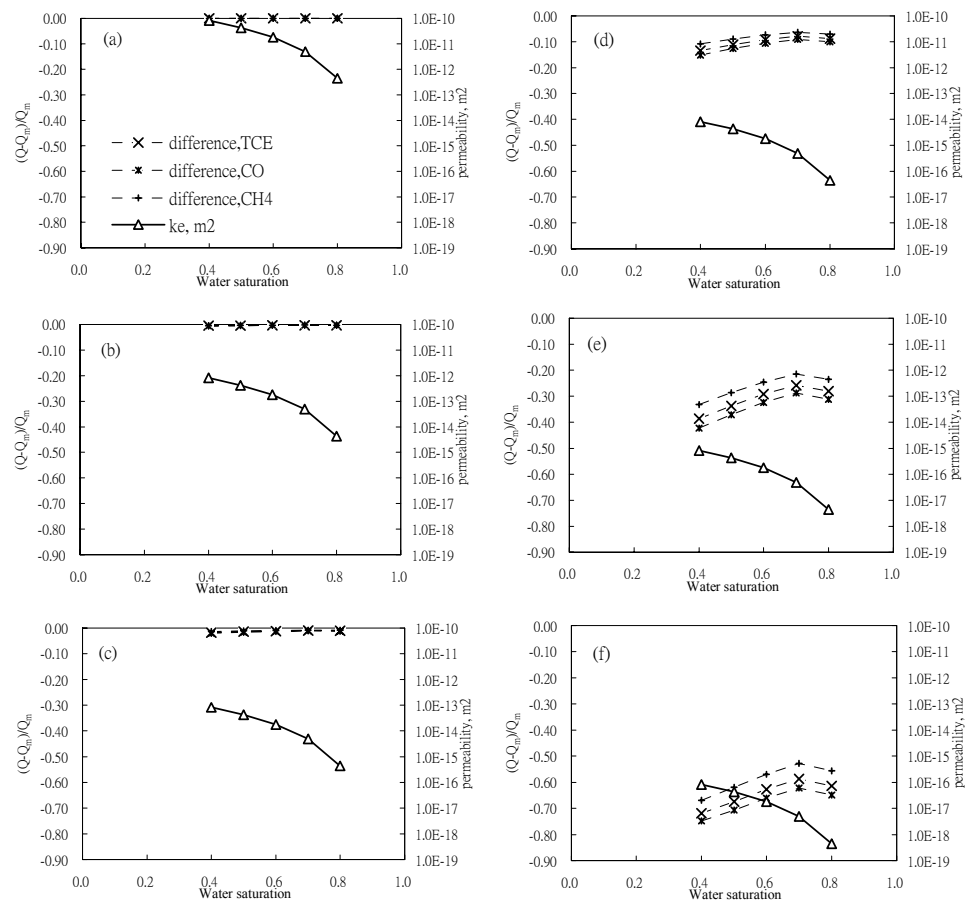

Figure 1: Comparison of values of the diffusibility and the obstruction factors for gas diffusion systems with a dry soil permeability of: (a) $10^{-10} \mathrm{~m}^{2}$; (b) $10^{-12} \mathrm{~m}^{2}$; (c) $10^{-13} \mathrm{~m}^{2}$; (d) $10^{-14} \mathrm{~m}^{2}$; (e) $10^{-15} \mathrm{~m}^{2}$; (f) $10^{-16} \mathrm{~m}^{2}$, at different water saturation levels 
Eqn (7) is also used to evaluate the diffusibility factor based on the experimental data given in articles by assuming that the corresponding diffusion experiments all involve a dilute diffusing gas. Results were compared to the diffusibility values provided in the articles as shown in figure 2. Values of the obstruction factor in eqn (7) for $[4,28,31]$ were calculated based on the correlation provided in [1] and for the others are based on [18]. Soil permeability values for the tested soil cores in these experiments were not provided in the referred articles. Hence, they were determined by fitting the diffusibility values obtained from eqn (7) to the ones provided in these articles. The permeability values obtained by the fitting are in the range between $10^{-13}$ and $10^{-20} \mathrm{~m}^{2}$, which is consistent with the permeability range for soils having high silt and clay contents. In this permeability range the difference between the diffusibility and obstruction factors is substantial as shown in figure 1. This implies that soil permeability (or the Knudsen diffusion coefficient) is an important factor affecting gas diffusion in these experiments and should be considered in the diffusibility factor. Permeabilities of the soil cores in these diffusion experiments are also evaluated according to eqn (8) and compared to the permeability values obtained by the fitting. Figure 3 shows that the permeability values obtained with the empirical equation are generally greater than the ones obtained by the fitting, implying that soil permeability is overpredicted by eqn (8).

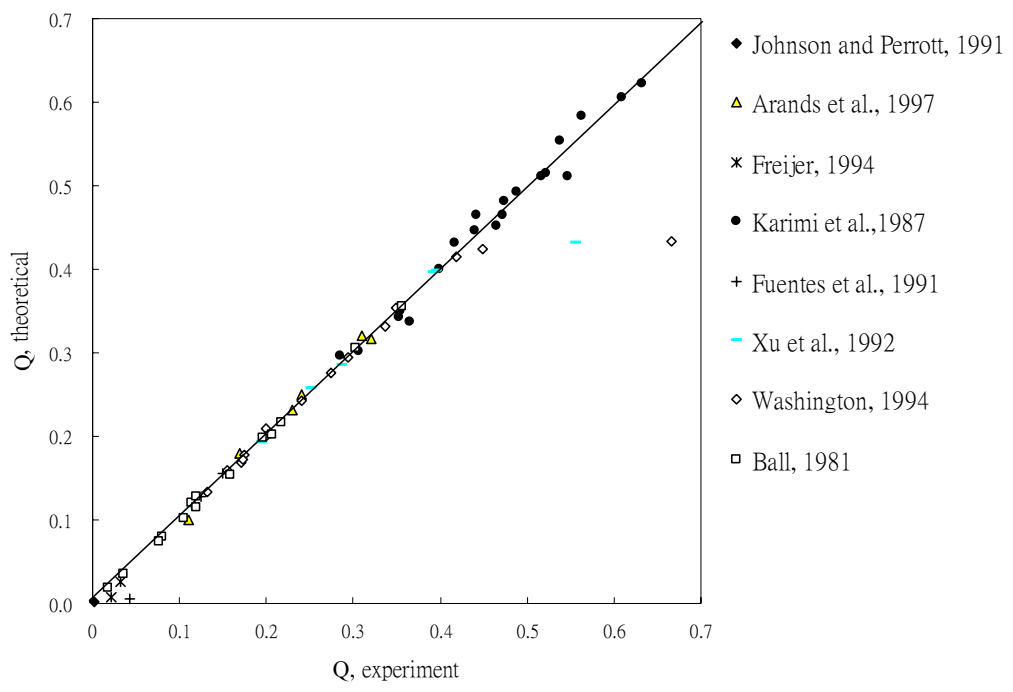

Figure 2: Comparison of the diffusibility factors calculated with eqn (7) and those given in the articles. 


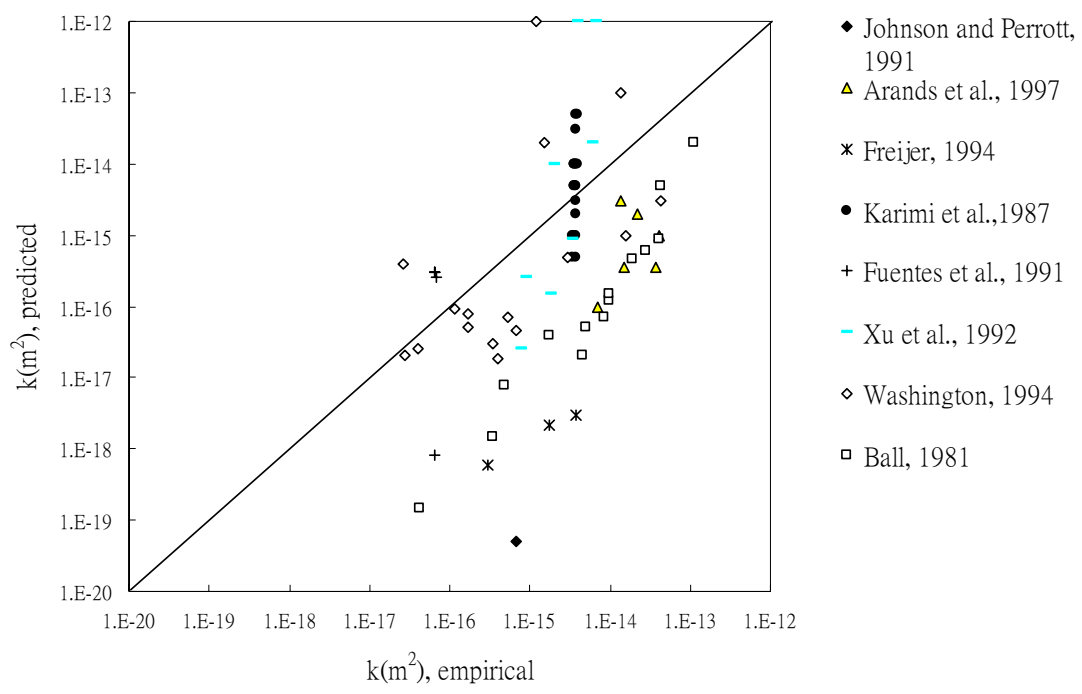

Figure 3: Comparison of the permeability values determined from fitting the diffusibility data and the ones calculated with eqn (8).

\section{Conclusions}

According to the study result, some conclusions are made as follows:

(1) The diffusibility factor for Fick's law of diffusion is identical to the obstruction factor defined in the DGM equations for diffusion systems with dry soil permeabilities greater than $10^{-13} \mathrm{~m}^{2}$ at water saturation levels ranging from 0.4 to 0.8 . However, for systems with lower dry soil permeabilities at any saturation, these two factors are not identical.

(2) Use of different gas components in diffusion experiments can obtain different diffusibility results for systems with dry soil permeabilities less than $10^{-13} \mathrm{~m}^{2}$ even though diffusing gases are dilute in the systems.

(3) Incorporation of the Knudsen diffusion coefficient into the diffusibility factor helps to adequately assess gas diffusion in soil systems. For this assessment, the obstruction factor and soil permeability should be determined independently through two-component gas diffusion and single gas flow experiments on the same soil sample. The Knudsen effect on the diffusibility factor is demonstrated through comparing results obtained with this diffusibility to the measured diffusibilities for 72 soil cores, which all have high silt and clay contents, given in papers. 


\section{Acknowledgements}

Funding of this research was provided by the National Science Council in Taiwan.

\section{References}

[1] Abu-El-Sha'r, W. \& Abriola, L.M., Experimental assessment of gas transport Mechanisms in natural porous media: parameter evaluation. Water Resources Research. 33(4), pp. 505-516, 1997.

[2] Amali, S., Rolston, D. E. \& Yamaguchi, T., Transient multicomponent gas-phase transport of volatile organic chemicals in porous media. $J$. Environ. Qual., 25, pp. 1041-1047, 1996.

[3] Arands, R.; Lam, T., Massry, I., Berler, D.H., Muzzio, F. J. \& Kosson, D.S., Modeling and experimental validation of volatile organic contaminant diffusion through an unsaturated soil. Water Resources Research 33(4), pp. 599-609, 1997.

[4] Ball, B. C., Modeling of soil pores as tubes using gas permeabilities, gas diffusivities and water release. Journal of Soil Science, 32, pp. 465-481, 1981.

[5] Bartelt-Hunt, A. L. \& Smith, J. A., Measurement of effective air diffusion coefficients for trichloroethene in undisturbed soil cores. Journal of Contaminant Hydrology, 56, pp. 193-208, 2002.

[6] Brugeman, D.A.G., Ann. Phys., 24, 636, 1935.

[7] Cunningham, R.E. \& Williams, R.J., Diffusion in gases and porous media, New York: Plenum, 1980.

[8] Currie, J. A., Movement of gases in soil respiration. Topic in Sorption and Transport Processes in Soils, 37, Monogr. Soc. Chem. Ind., pp. 152-171, Rothamsted Exp. Stn., Harpenden, England, 1970.

[9] Fen, C.-S. \& Abriola, L.M., A comparison of mathematical model formulations for organic vapor transport in porous media. Advances in Water Resources, 27(10), pp.1005-1016, 2004.

[10] Freijer, J. I., Calibration of jointed tube model for the gas diffusion coefficient in soils. Soil Sci. Soc. Am. J., 58, pp. 1067-1076, 1994.

[11] Fuentes, H. R., Polzer, W. L. \& Smith, J. L., Laboratory measurements of diffusion coefficients for trichloroethylene and orthoxylene in undisturbed tuff, J. Environ. Qual., 20, pp. 215-221, 1991.

[12] Jaynes, D.B. \& Rogowski, A.S., Applicability of Fick's law to gas diffusion, Soil Sci. Soc. Am. J., 47, pp.425-430, 1983.

[13] Johnson, R. L. \& Perrott, M., Gasoline vapor transport through a highwater-content soil. Journal of Contaminant Hydrology, 8, pp.317-334, 1991.

[14] Jury, W.A., Russo, D., Streile, G. \& Abd, A.E., Evaluation of volatilization by organic chemicals residing below the soil surface. Water Resour. Res., 26(1), pp. 13-20, 1990. 
[15] Karimi, A. A., Farmer, W. J. \& Cliath, M. M., Vapor-phase diffusion of benzene in soil. J. Environ. Qual., 16(1), pp. 38-43, 1987.

[16] Kozak, J.A., Reeves, H.W., Lewis, B. A., Modeling radium and radon transport through soil and vegetation. J. of Con. Hyd., 66, pp. 179-200, 2003.

[17] Lahvis, M.A., Baehr, A.L. \& Baker, R.J., Evaluation of volatilization as a natural attenuation pathway for MTBE, Ground Water, 42(2), pp. 258-266, 2004.

[18] Millington, R. J. \& Quirk, J. P., Permeability of porous media. Trans. Faraday Soc., 57, pp.1200-1207, 1961.

[19] Moldrup, P., Olesen, T., Schjonning, P., Yamaguchi, T. \& Rolston, D.E., Predicting the gas diffusion coefficient in undisturbed soil from soil water characteristics. Soil Sci. Soc. Am. J., 64, pp. 94-100, 2000.

[20] Nastev, M, Therrirn, R., Lefebvre, R. \& Gelinas, O., Gas production and migration in landfills and geological materials. J. of Conta. Hydro., 52, pp. 187-211, 2001.

[21] Neale, G. H. \& Nader, W. K., A. I.Ch.E. Journal, 19,112, 1973

[22] Penman, H. L., Gas and vapour movement in the soil, I, the diffusion of vapour through porous solids. J. Agric. Sci., 30, pp. 437-462, 1940.

[23] Reinecke, S.A. \& Sleep, B.E., Knudsen diffusion, gas permeability, and water content in an unsaturated porous medium. Water Resour. Res., 38(12), pp. 1280-1294, 2002.

[24] Schaefer, C. E., Arands, R. R., van der Sloot, H. A. \& Kosson, D. S., Modeling of the gaseous diffusion coefficient through unsaturated soil systems. Journal of Contaminant Hydrology, 29, pp. 1-21, 1997.

[25] Thorstenson, D. C. \& D. W. Pollock, Gas transport in unsaturated zones: Multicomponent systems and the adequacy of Fick's law. Water Resources Research, 25 (3), pp. 477-507, 1989.

[26] Troeh, F.R., Jabro, J. D. \& Kirkham, D. Geoderma, 27, 239, 1982.

[27] Van Duin, R.H.A., Over De. Invloed Van Grondbwerking Op Het Transport Vvan Warmte. Water En Lucht, Verst Landbouwk Orderz 62.7, Staatsdrukkerij, The Hague, 81pp, 1956.

[28] Washington, J.W., Rose, A. W., Ciolkosz, E. J. \& Dobos, R. R., Gaseous diffusion and permeability in four soil profiles in central Pennsylvania, Soil Science, 157(2), pp. 65-76,1994.

[29] Webb, W. S. \& Pruess, K., The use of Fick's law for modeling trace gas diffusion in porous media. Transport in Porous Media, 51, pp. 327-341, 2003.

[30] Weers, A.H., Freijer, J.I. \& Bouten, W., Modeling the gas diffusion coefficient in analogy to electrical conductivity using a capillary model. Soil Sci. Soc. Am. J. 64, pp. 527-532, 2000.

[31] Xu, X., Nieber, J.L. \& Gupta, S.C., Compaction effect on the gas diffusion coefficient in soils. Soil Sci. Soc. Am. J. 56, pp. 1743-1750, 1992. 